\title{
1 Incorporation of metabolically stable ketones into a small 2 molecule probe to increase potency and water solubility.
}

3 Marie-Helene Larraufie, ${ }^{a}$ Wan Seok Yang, ${ }^{a}$ Elise Jiang, ${ }^{b}$ Ajit G. Thomas, ${ }^{c}$ Barbara S. Slusher,,${ }^{c, d}$ and Brent R.

4 Stockwell*a,b,e

$5 \quad{ }^{a}$ Department of Biological Sciences, Columbia University, 550 West 120th Street, 1208 Northwest Corner Building, 6 MC 4846 New York, New York 10027, United States.

7 bepartment of Chemistry, Columbia University, New York, New York 10027, United States.

$8 \quad{ }^{\mathrm{C}}$ Brain Science Institute, Johns Hopkins Medicine, Baltimore, United States.

9 dDepartment of Neurology, Johns Hopkins Medicine, Baltimore, United States

10 eHoward Hughes Medical Institute, Columbia University, New York, New York 10027, United States.

12 ABSTRACT: Introducing a reactive carbonyl to a scaffold that does not otherwise have an electrophilic

13 functionality to create a reversible covalent inhibitor is a potentially useful strategy for enhancing 14 compound potency. However, aldehydes are metabolically unstable, which precludes the use of this strategy for compounds to be tested in animal models or in human clinical studies. To overcome this limitation, we designed ketone-based functionalities capable of forming reversible covalent adducts,

17 while displaying high metabolic stability, and imparting improved water solubility to their pendant scaffold. We tested this strategy on the ferroptosis inducer and experimental therapeutic erastin, and observed substantial increases in compound potency. In particular, a new carbonyl erastin analog, termed IKE, displayed improved potency, solubility and metabolic stability, thus representing an ideal candidate future in vivo cancer therapeutic applications.

There is growing awareness that compounds with optimal properties in terms of selectivity and potency might be obtained by combining the distinct properties of covalent and non-covalent ligands. ${ }^{1}$

Page | 1

(C) 2015. This manuscript version is made available under the Elsevier user license http://www.elsevier.com/open-access/userlicense/1.0/ 
1 Practically, this often means functionalizing a scaffold optimized for specific complementarity to a target

2 with a carefully tuned reactive moiety, to create a targeted covalent inhibitor. A powerful way to

3 mitigate the risks of off-target reactivity is to use a reactive moiety that forms a reversible covalent bond

4 with proteins. In addition to increased selectivity, reversible covalent inhibitors may limit the risk of

5 haptenization and immunogenicity associated with the formation of circulating irreversibly modified

6 protein adducts. ${ }^{2,3,4,5}$

7 An important class of reversible covalent inhibitors are aldehyde-based compounds capable of forming

8 an imine with a lysine side chain in the binding site of a target protein. Recent examples include the IRE1

9 endonuclease inhibitors $4 \mu 8 c^{6}$ and STF083010, ${ }^{7}$ a 20 S proteasome inhibitor $^{8}$ and the tau fibrillization

10 inhibitor oleocanthal. ${ }^{9}$ While such reversible covalent inhibitors have mostly been discovered

11 serendipitously, the rational design of selective imine-forming electrophiles may be feasible via the

12 identification of accessible lysine residues and simulation-based pKa calculations. ${ }^{10}$ Indeed, it appears

13 from recent literature that lysines buried in hydrophobic pockets, such as those targeted by small-

14 molecules, have their pKa downshifted by 3 to 5 units compared to solvent-exposed lysines. ${ }^{11,12}$ This

15 feature would favor the deprotonation of buried lysine side chains and increased nucleophilicity,

16 facilitating selective attack on a proximal carbonyl functionality.

17 Introducing an aldehyde moiety to an otherwise non-covalent ligand to convert it into a reversible

18 covalent inhibitor may thus be feasible. However, the low metabolic stability of aldehydes generally

19 precludes their utilization for in vivo applications. Indeed, mammals have evolved a range of enzymes to

20 transform aldehydes into the corresponding alcohols (aldoketo reductases) and carboxylic acids

21 (aldehyde dehydrogenases/cytochromes P450s). ${ }^{13}$ No carbonyl moieties capable of forming imines with

22 Iysine side chains have been described that are also metabolically stable. Described herein is the design 
1 and synthesis of such functionalities, with optimized metabolic stability and aqueous solubility, and their

2 incorporation into the scaffold of the small molecule ferroptosis probe erastin.

3 We previously showed that erastin induces preferential lethality in engineered human fibroblasts

4 overexpressing oncogenic HRAS. ${ }^{14}$ Erastin induces an oxidative form of non-apoptotic cell death, termed

5 ferroptosis, ${ }^{15}$ that is regulated by glutathione peroxidase $4 .{ }^{16}$ Erastin (Figure 1, ERA) exhibits modest

6 water solubility $(0.086 \mathrm{mM})$ and potency $\left(\mathrm{GI}_{50}=1.7 \mu \mathrm{M}\right.$ in $\mathrm{HRAS}^{\mathrm{G} 12 \mathrm{~V}}$-overexpressing tumorigenic cells

7 BJeLR), precluding its use in vivo. Introducing a piperazine moiety on the meta position of the aniline

8 ring of erastin enhances the water solubility of the scaffold. This piperazine erastin analog (Figure 1, PE)

9 displayed significant activity in a tumor prevention model using nude mice injected with NRAS mutant

10 human HT-1080 fibrosarcoma calls, in which PE was injected before tumors became established. ${ }^{16}$

11 However, PE had a limited effect on the growth of already established tumors, perhaps due to its

12 moderate potency. Replacing the piperazine moiety with an aldehyde functionality (Figure $1, A E$ )

13 allowed a substantial increase in potency, albeit at the expense of metabolic stability and solubility. ${ }^{16}$

14 Nevertheless, this large increase in potency brought about by the introduction of an aldehyde moiety

15 suggested that a lysine side chain in the vicinity of the binding site was susceptible to imine formation.

16 We consequently endeavored to design carbonyl-containing analogs with an optimal balance between

17 reactivity, stability and solubility, and tested the consequences of their incorporation into the erastin

18 scaffold. Using this strategy, we identified erastin analogs with metabolically stable electrophilic

19 ketones, and improved potency and metabolic stability compared to erastin and PE. 


\section{Previous work:}<smiles>CCOc1ccccc1-n1c(C(C)N2CCN(C(=O)COc3ccc(C)cc3)CC2)nc2ccccc2c1=O</smiles>

ERA

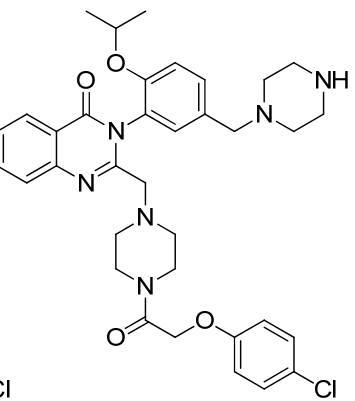

PE

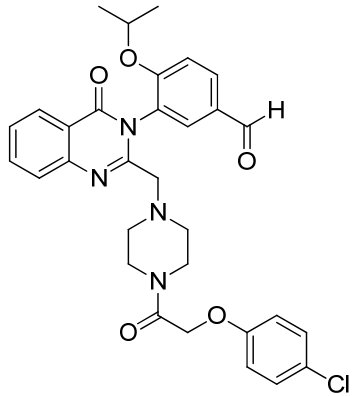

$\mathrm{AE}$

BJeLR GI $\mathrm{S}_{50}=1.7 \mu \mathrm{M}$ $\mathrm{T}_{1 / 2}$ microsome $<5 \mathrm{~min}$ BJeLR GI $50=0.9 \mu \mathrm{M}$ $\mathrm{T}_{1 / 2}$ microsome $=55 \mathrm{~min}$

BJeLR GI $50=8 \mathrm{nM}$ Solubility $($ water $)=0.09 \mathrm{mM}$ Solubility $($ water $)=1.4 \mathrm{mM}$ Solubility $($ water $)=0.08 \mathrm{mM}$

\section{I his work:}

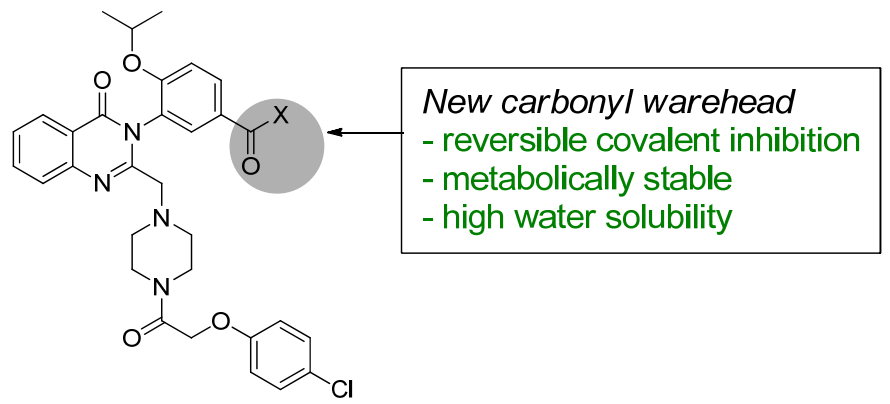

Figure 1. Structure of erastin analogs (top) and design of analogs (bottom) with improved properties.

$\alpha$-Substituted ketones can form imines with lysine side chain. In order to define the structural

4 requirements for facile imine formation, we compared the reactivity of aryl aldehydes and $\alpha$-substituted

5 aryl ketones towards a simple model of lysine side chain. Our idea was to use $n$-butyl amine to mimic the reactive side chain of lysine and determine whether this moiety could react with $\alpha$-substituted ketones

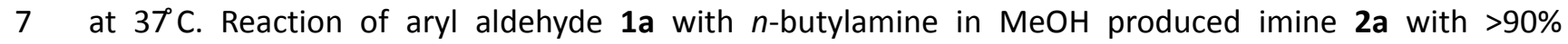

8 conversion after $24 \mathrm{~h}$ at $37^{\circ} \mathrm{C}$. High conversion to the imine adduct were also obtained with methyl ketone $\mathbf{1 b}$ and $\alpha$-fluoromethyl ketone $\mathbf{1 c}$. We observed that when the methyl ketone was $\alpha$-substituted with heterocycles, the formation of the imine adduct could still be observed, although the conversion was lower under these conditions. We tested in total a set of 9 carbonyl-containing moieties after $24 \mathrm{~h}$ at 
$137{ }^{\circ} \mathrm{C}$ (Figure 2). We found that $\alpha$-substituted ketones could be used as an electrophile for imine

2 formation, and the relative reactivity of each carbonyl moiety was determined.

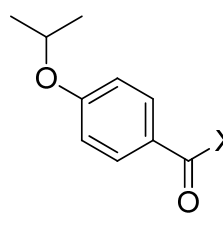

1a-i

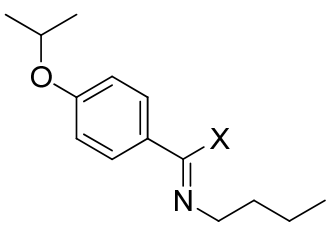

2a-i

\begin{tabular}{cccc}
\hline Probe, $\mathrm{X}$ & Imine/SM ratio & Probe, $\mathrm{X}$ & Imine/SM ratio \\
\hline $\mathbf{1 a}, \mathrm{X}=\mathrm{H}$ & $10 / 1$ & $\mathbf{1 b}, \mathrm{X}=\mathrm{Me}$ & $3 / 1$ \\
\hline $\mathbf{1 c}, \mathrm{X}=\mathrm{CH}_{2} \mathrm{~F}$ & $1 / 1$ & & $1 / 2.5$ \\
\hline $\mathbf{1 d}, \mathrm{X}=$ & $1 / 1.2$ & $\mathbf{1 e}, \mathrm{X}, \mathrm{X}$ \\
\hline $\mathbf{1 f}, \mathrm{X}=$
\end{tabular}

4

5

Figure 2. Comparative reactivities of carbonyl moities with n-butylamine. Carbonyl derivative 1a-i were reacted with $n$-butylamine in $\mathrm{MeOH}$, and the imine adduct to starting material ratio (imine/SM) was measured after $24 \mathrm{~h}$ by treating the mixture with $\mathrm{NaBH}_{3} \mathrm{CN}$, and isolating the corresponding amine and SM.

Synthesis of carbonyl erastin analogues. We endeavored to develop an efficient synthetic strategy to incorporate reactive carbonyl moieties in the meta position of the aniline-derived moiety of erastin, as a model for incorporating such functionalities on aromatic regions of small molecules generally. A methyl ketone derivative, KE (Figure 3, entry 4), was synthesized in 5 steps (overall yield 21\%) from commercially available 4-hydroxy-3-nitroacetophenone (see supporting information). Similarly, $\alpha$-fluoro ketone erastin (FKE) and trifluoroketone erastin (TFKE) (Figure 3, entries 5 and 6) were prepared in 6 steps from 4hydroxy-3-nitroacetophenone and 3-nitrophenol respectively (see supporting information). Finally, we 
1 synthesized morpholine ketone erastin (MKE), N-methylpiperazine ketone erastin (MPKE), N-

2 allylpiperazine ketone erastin (APKE), N-p-methoxybenzylpiperazine ketone erastin (PMB-PKE),

3 piperazine ketone erastin (PKE) and imidazole ketone erastin (IKE) (Figure 3, entries 7-12) using the

4 general synthetic route depicted in Scheme 1 for APKE and PKE.
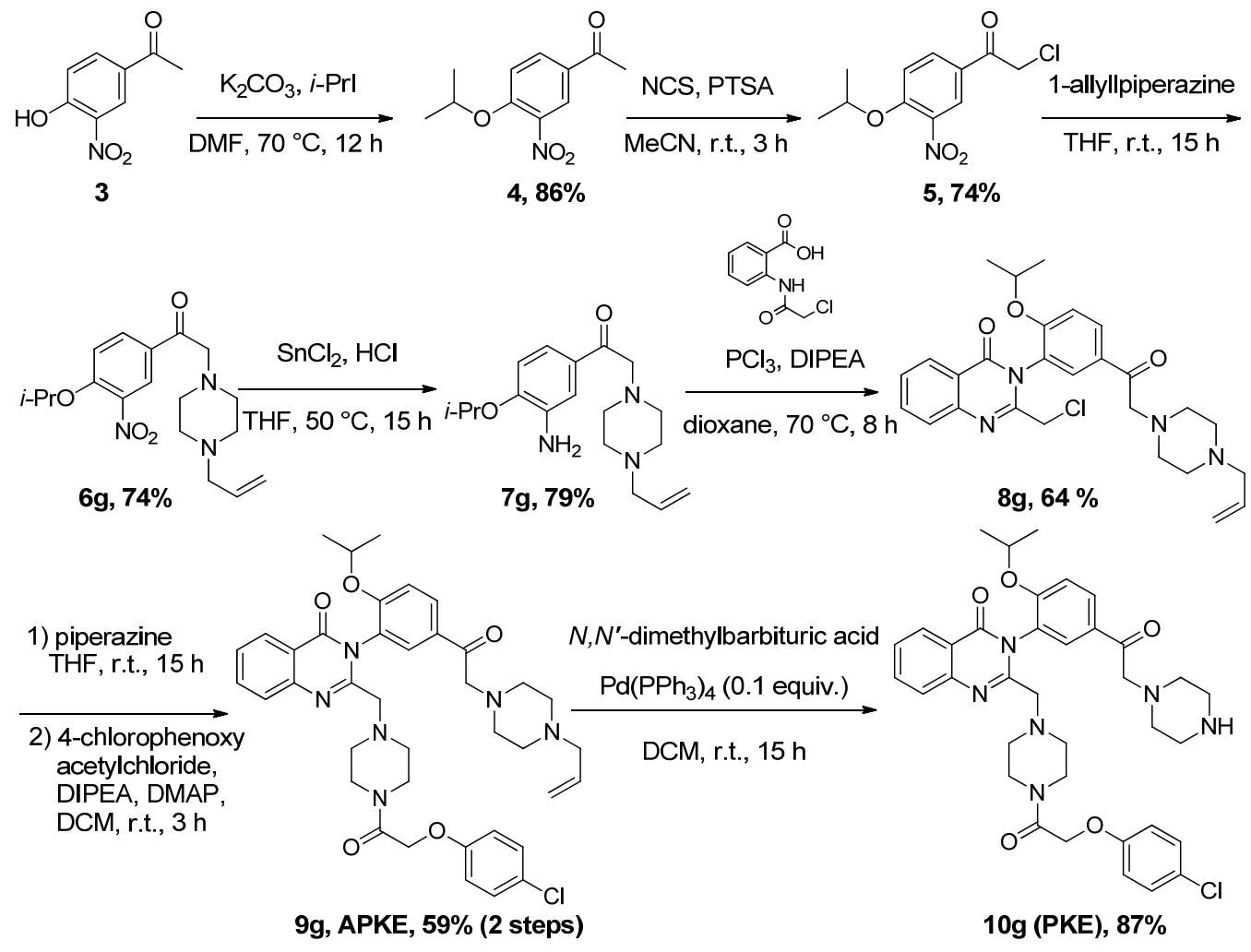

Scheme 1. Synthetic route to piperazine ketone erastin (PKE).

7 Carbonyl erastin analogues are potent system xc- inhibi-tors. We previously demonstrated that erastin

8 induces ferroptosis via the inhibition of cystine uptake through the cystine-glutamate antiporter (system

$\left.9 \mathrm{x}_{c}^{-}\right) \cdot{ }^{15}$ The ten ketone erastin analogs were evaluated in a glutamate release assay, which reports on system $x_{c}^{-}$activity. Human astrocytoma cells (CCF-STTG1) were used, which contain the system $x_{c}{ }^{-17}$

11 Following a $2 \mathrm{~h}$ incubation period, glutamate released into the medium was detected fluorometrically

12 using glutamate oxidase, horseradish peroxidase and Amplex UltraRed. Dramatic improvement of the 
1 half-maximal inhibitory constants $\left(\mathrm{IC}_{50}\right)$ for system $\mathrm{x}_{c}^{-}$inhibition was observed for all ketone analogs,

2 compared to erastin and PE (Figure 3). Several analogs, such as MKE (Figure 3, entry 7, IC $50=10 \mathrm{nM}$ ) and 3 IKE (Figure 3, entry 12, IC $50=30 \mathrm{nM}$ ) even had improved potency over aldehyde erastin (Figure 3, entry $\left.4 \quad 3, \mathrm{IC} \mathrm{C}_{50}=60 \mathrm{nM}\right)$.

6

7

8

9

列

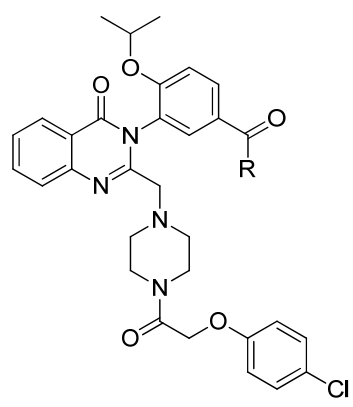

.

(8)

Carbonyl erastin analogs exhibit selective lethality in BJ-derived tumorigenic cells expressing

oncogenic HRAS. Encouraged by this improved potency in the system xc- assay, we tested the potency and genotype-selective lethality of the ketone analogs in BJ-derived cell lines. Compounds were tested in 4 isogenic cell lines: HRAS ${ }^{G 12 V}$-overexpressing tumorigenic cells (BJeLR, DRD) and non-transformed isogenic cells without mutant HRAS expression (BJeHLT, BJeH). We observed that all the analogs except MPKE and APKE were preferentially lethal to BJeLR and DRD cells compared to BJeH and BJeHLT cells (Figure 4A and Figure S1). In addition, AE, KE, FKE, PKE and IKE demonstrated low nanomolar potencies in the HRAS ${ }^{G 12 V_{-}}$-overexpressing cell lines. 


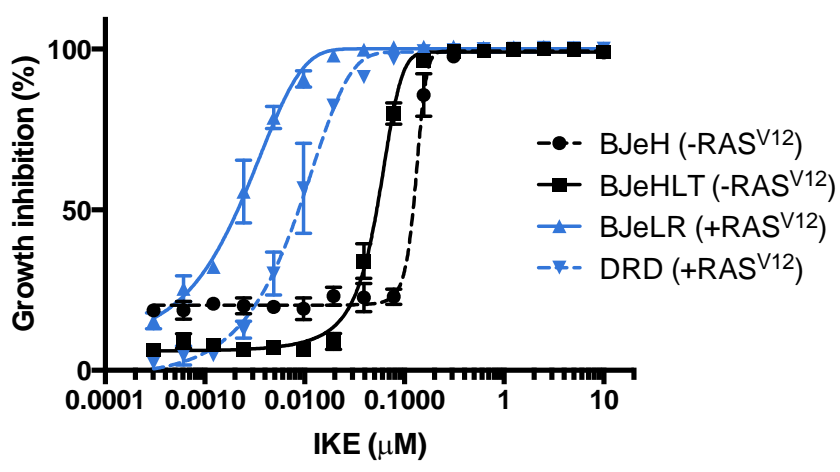

\begin{tabular}{cccc}
\hline Entry & Analogue & IC50 BJeLR (nM) & Selectivity (IC50BJeH/IC50BLeLR) \\
\hline 1 & Erastin & 625 & 4.6 \\
\hline 2 & PE & 300 & 3 \\
\hline 3 & AE & 8 & 15 \\
\hline 4 & KE & 65 & 15 \\
\hline 5 & FKE & 51 & 8.3 \\
\hline 6 & TFKE & 210 & 8 \\
\hline 7 & MKE & 427 & 5 \\
\hline 8 & MPKE & 474 & 1.9 \\
\hline 9 & APKE & 195 & 1.5 \\
\hline 10 & PMB-PKE & 13 & 13 \\
\hline 11 & PKE & 12 & 32 \\
\hline 12 & IKE & 3 & 42 \\
\hline
\end{tabular}

4 Figure 4. Selective lethality of carbonyl erastin analogs in BJ-derived cell lines. (A) Growth inhibition of BJ cell lines after $24 \mathrm{~h}$ treatment with IKE. (B) Erastin analog poten-cies and selectivities in BJ cells.

7 human fibrosarcoma cell line. Treatment of HT-1080 cells for $48 \mathrm{~h}$ with either PKE or IKE resulted in

8 complete growth inhibition. We determined that IKE ( $\left.\mathrm{II}_{50}=310 \mathrm{nM}\right)$ was almost twice as potent as PKE $9 \quad\left(\mathrm{Gl}_{50}=550 \mathrm{nM}\right)$ in this cell line (see Figure S2). 
1 Two carbonyl erastin analogues, IKE and PKE display high metabolic stability. Our rationale for the

2 design of new carbonyl warheads was that the substitution of the methylene alpha to the ketone

3 carbonyl with polar bulky moieties would both enhance the water solubility of the analogs and hamper

4 metabolic degradation (e.g. reduction) of that sensitive position by cytochrome P450 enzymes. We

5 assessed experimentally the half-lives of the eight most potent analogues in mouse liver microsomes.

6 The carbonyl erastin analogs were incubated at $37 \stackrel{\circ}{\circ}$ for up to 45 minutes in potassium phosphate

7 buffer $(\mathrm{pH}$ 7.4) containing mouse liver microsomal proteins and an NADPH-generating system. At 0,5 ,

815,30 and 45 min intervals, aliquots were removed and analyzed by LC-MS/MS to determine the half-life

9 and the intrinsic clearance of each compound (see Figure S3). As predicted, both the aldehyde analog

10 (figure 5, AE) and the methyl ketone analog (Figure 5, KE) were rapidly metabolized and had half-lives $<5$

11 min. Several other carbonyl erastin analogs (Figure 5: FKE, MPKE, APKE, PMB-PKE) displayed medium to

12 poor metabolic stability. On the other hand, to our delight, two carbonyl erastin analogues, piperazine

13 ketone erastin (Figure 5, PKE, $\mathrm{T}_{1 / 2}>90 \mathrm{~min}$ ) and imidazole ketone erastin (Figure 5, IKE, $\mathrm{T}_{1 / 2}=79 \mathrm{~min}$ )

14 demonstrated exceptionally high metabolic stability that even surpassed that of piperazine erastin,

15 which does not possess a carbonyl functionality (Figure 5, PKE, $\mathrm{T}_{1 / 2}=55 \mathrm{~min}$ ).

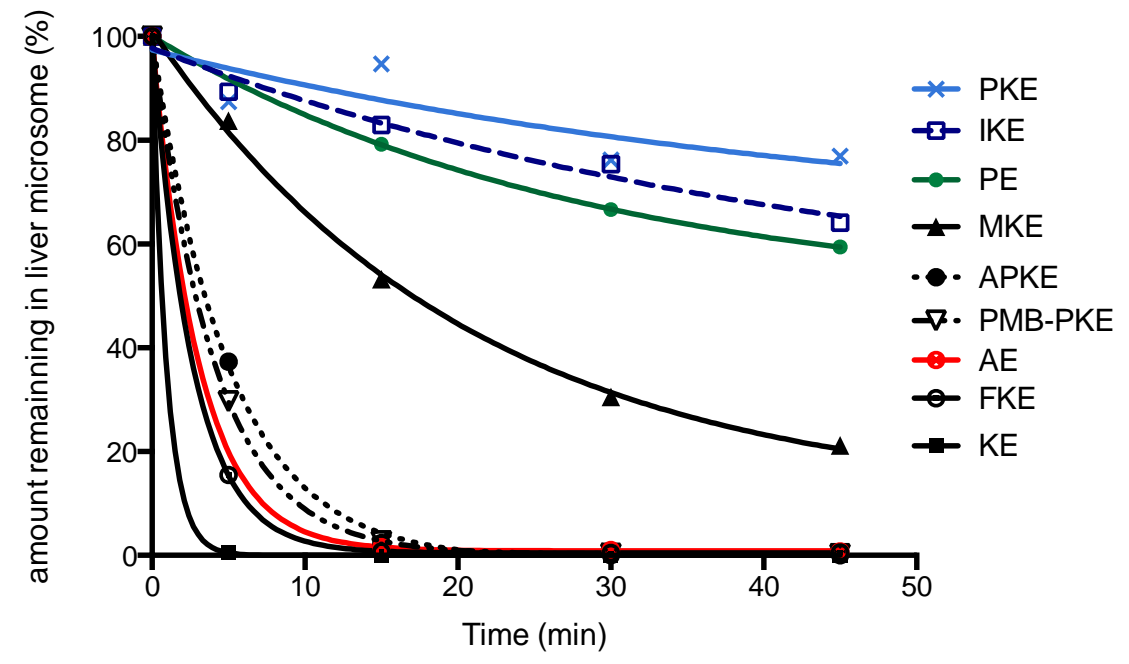

17 
Figure 5. Metabolic stability of carbonyl erastin analogs in mouse liver microsomes.

2 In addition, we assessed the plasma stability and PK profile of IKE and PKE in mice. Stability of PKE and

3 IKE in mouse plasma was determined by dissolving each compound in the mouse plasma at $500 \mathrm{ng} / \mathrm{mL}$

4 concentration followed by incubation at $37^{\circ} \mathrm{C}$. Both PKE and IKE remained stable up until 120 min under

5 this condition (see SI-Figure 2).

7 The design of covalent inhibitors has recently attracted renewed interest, with several covalent drug 8 candidates now progressing to late-stage clinical trials. ${ }^{1}$ This renaissance stems in part from the greater 9 appreciation that low doses of highly potent targeted covalent inhibitors have few off-target effects, ${ }^{18,19}$ 10 and that reversible covalent inhibitors are even less likely to exhibit immunotoxicity. ${ }^{20}$

11 Although most current covalent drugs were discovered through serendipity, there is now an interest in 12 the explicit design of targeted covalent inhibitors. The design of such compounds involves identifying a non-covalent scaffold with high potency, followed by functionalization with moieties capable of forming covalent interactions. ${ }^{21,22,23,24,25}$ Here, we reported the design of ketone moieties able to engage in 15 reversible imine formation with lysine residues. In contrast to an aldehyde, these optimized ketone moieties are metabolically stable and can enhance the solubility of the pendant scaffold. They are thus

17 promising functionalities to append to otherwise non-covalent inhibitors in order to improve potency 18 and solubility without compromising metabolic stability. We demonstrated the validity of this approach 19 by functionalizing the small molecule probe erastin with carbonyl warheads. The carbonyl erastin analogs demonstrated clear increased potency as system $x_{c}^{-}$inhibitors, compared to erastin and 21 piperazine erastin. In addition, two ketone erastin analogs, PKE and IKE, displayed low nanomolar 22 potency and selective lethality towards BJ-derived tumorigenic cells expressing oncogenic HRAS. Use of 23 these ketone moieties has resulted in the creation of a ketone erastin analogue, IKE, with optimized 
1 properties for in vivo use, which represents a decisive milestone in the development of therapeutic

2 ferroptosis inducers. In addition, this successful application highlights the potential of these rationally

3 designed carbonyl warhead for improving compounds for in vivo applications.

4

5

6

8

\section{ASSOCIATED CONTENT}

Supporting Information.

\section{AUTHOR INFORMATION}

Corresponding Author: For additional information, contact Brent R. Stockwell at bstockwell@columbia.edu.

Funding Sources:The authors have no competing financial interests. This research was supported by grants to BRS (NIH 5R01CA097061, 5R01GM085081, and R01CA161061). BRS is an Early Career Scientist of the Howard Hughes Medical Institute.

\section{ACKNOWLEDGMENT}

We thank Dr. John Decatur and the Columbia Chemistry NMR core facility (NSF grant CHE 0840451 and NIH grant 1S10RR025431-01A1) for assistance in compound synthesis.

\section{REFERENCES}

(1) Singh, J.; Petter, R. C.; Baillie, T. A.; Whitty, A. Nat. Rev. Drug Disc. 2011, $10,307$.

(2) Serafimova, I. M.; Pufall, M. A.; Krishnan, S.; Duda, K.; Cohen, M. S.; Maglathlin, R. L.; Mcfarland, J. M.; Miller, R. M.; Frödin, M.; Taunton, J. Nat. Chem. Biol. 2012, 8, 471.

(3) Miller, R. M.; Paavilainen, V. O.; Krishnan, S.; Serafimova, I. M.; Taunton, J. J. Am. Chem. Soc. 2013, 135, 5298.

(4) Lee, C.-U.; Grossmann, T. N. Angew. Chem. Int. Ed. 2012, 51, 8699.

(5) Patch, R. J.; Searle, L. L.; Kim, A. J.; De, D. Y. D.; Zhu, X. Z.; Askari, H. B.; ONeill, J. C.; Abad, M. C.; Rentzeperis, D.; Liu, J. Y.; Kemmerer, M.; Lin, L.; Kasturi, J.; Geisler, J. G.; Lenhard, J. M.; Player, M. R.; Gaul, M. D. J. Med. Chem. 2011, 54, 788.

(6) Cross, B. C. S.; Bond, P. J.; Sadowski, P. G.; Jha, B. K.; Zak, J.; Goodman, J. M.;Silverman, R. H.; Neubert, T. A.; Baxendale, I. R.; Ron, D.; Harding, H. P. Proc. Natl. Acad. Sci. U. S. A. 2012, 109, E869. 
(7) Papandreou, I.; Denko, N. C.; Olson, M.; Van Melckebeke, H.; Lust, S.; Tam, A.; Solow-Cordero, D. E.; Bouley, D. M.; Offner, F.; Niwa, M.; Koong, A. C. Blood 2011, 117, 1311.

(8) Ma, Y.; Xu, B.; Fang, Y.; Yang, Z.; Cui, J.; Zhang, L.; Zhang, L. Molecules 2011, 16, 7551.

(9) Li, W.; Sperry, J. B.; Crowe, A.; Trojanowski, J. Q.; Smith III, A. B.; Lee, V. M.-Y. J. Neurochem. 2009, 110, 1339.

(10) Tomasio, S. M.; Harding, H. P.; Ron, D.; Cross, B. C. S.; Bond, P. J. Mol. BioSyst. 2013, 9, 2408.

(11) Barbas, C. F., III; Heine, A.; Zhong, G.; Hoffmann, T.; Gramatikova, S.; Bjornestedt, R.; List, B.; Anderson, J.; Stura, E. A.; Wilson, I. A.; Lerner, R. A. Science 1997, 278, 2085.

(12) Isom, D. G.; Castañeda, C. A.; Cannon, B. R.; Garcia-Moreno, E. B. Proc. Natl. Acad. Sci. U. S. A. 2011, $108,5260$.

(13) Conklin, D.; Prough, R.; Bhatanagar, A. Mol. BioSyst. 2007, 3, 136.

(14) Dolma, S.; Lessnick, S. L.; Hahn, W. C.; Stockwell, B. R. Cancer Cell 2003, 3, 285.

(15) Dixon, S. J.; Lemberg, K. M.; Lamprecht, M. R.; Skouta, R.; Zaitsev, E. M.; Gleason, C. E.; Patel, D. N.; Bauer, A. J.; Cantley, A. M.; Yang, W. S.; Morrison, B.; Stockwell B. R. Cell 2012, 149, 1060.

(16) Yang, W. S.; SriRamaratnam, R.; Welsch, M. E.; Shimada, K.; Skouta, R.; Viswanathan, V. S.; Cheah, J. H.; Clemons, P. A.; Shamji, A. F.; Clish, C. B.; Brown L. M.; Girotti, A. W.; Cornish, V. W.; Schreiber, S. L.; Stockwell, B. R. Cell 2014, 156, 317.

(17) Dixon, S. J.; Patel, D. N.; Welsch, M. E.; Skouta, R.; Lee, E. D.; Hayano, M.; Thomas, A. G.; Gleason, C. E.; Tatonetti, N. P.; Slusher, B. S.; Stockwell, B. R. eLife 2014, 3, e02523.

(18) Wu, H.; Wang, W.; Liu, F.; Weisberg, E. L.; Tian, B.; Chen, Y.; Li, B.; Wang, A.; Wang, B.; Zhao, Z.; McMillin, D. W.; Hu, C.; Li, H.; Wang, J.; Liang, Y.; Buhrlage, S. J.; Liang, J.; Liu, J.; Yang, G.; Brown, J. R.; Treon, S. P.; Mitsiades, C. S.; Griffin, J. D.; Liu, Q.; Gray, N. S. ACS Chem. Biol., 2014, 9, 1086.

(19) Lanning, B. R.; Whitby, L. R.; Dix, M. M.; Douhan, J.; Gilbert, A. M.; Hett, E.C.; Johnson, T. O.; Joslyn, C.; Kath, J.C.; Niessen, S.; Roberts, L. R.; Schnute, M. E.; Wang, C.; Hulce, J. J.; Wei, B.; Whiteley, L. O.; Hayward, M. M.; Cravatt, B. F. Nat. Chem. Biol. 2014, 10, 760.

(20) Maha, R.; Thomas, J. R.; Shafer, C. M. Bioorg. Med. Chem. Lett. 2014, 24, 33.

(21) Cohen, M. S.; Zhang, C.; Shokat, K. M.; Taunton, J. Science 2005, 308, 1318.

(22) Zhou, W.; Hur, W.; McDermott, U.; Dutt, A.; Xian, W.; Ficarro, S. B.; Zhang, J.; Sharma, S. V.; Brugge, J.; Meyerson, M. et al. Chem. Biol. 2010, 17, 285.

(23) Kwarcinski, F. E.; Fox, C. C.; Steffey, M. E.; Soellner, M. B. ACS Chem Biol. 2012,7, 1910.

(24) Ward, R. A. et al. J. Med. Chem. 2013, 56, 7025.

(25) Zhang, T. et al. Chem. Biol. 2012, 19, 140.
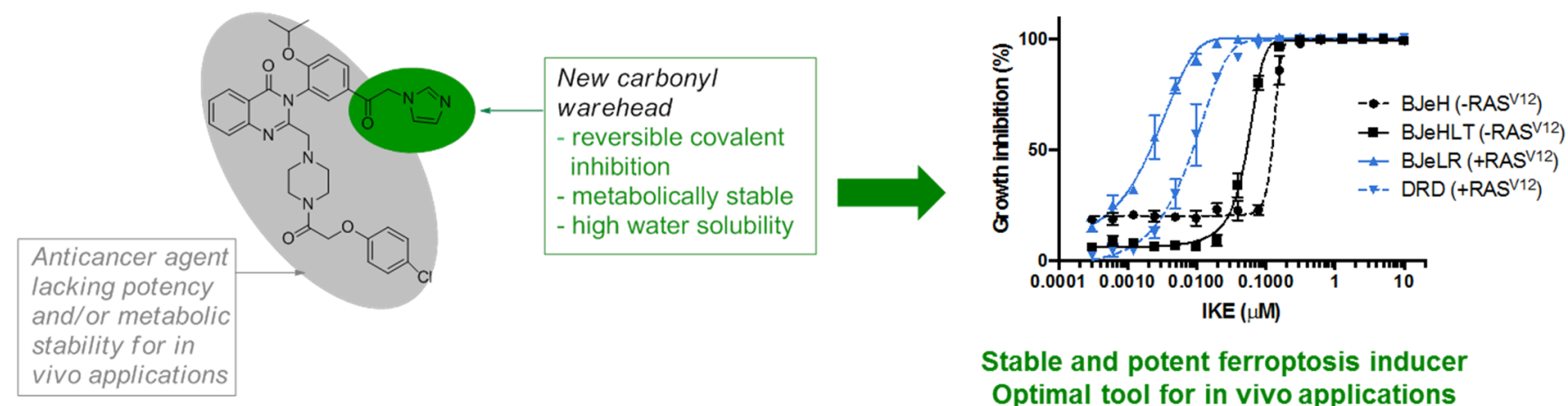

Stable and potent ferroptosis inducer Optimal tool for in vivo applications 